\title{
Actinobacillus species isolated from Japanese Thoroughbred racehorses in the last two decades
}

\author{
Eri UCHIDA-FUJII ${ }^{1) *}$, Hidekazu NIWA ${ }^{1)}$, Yuta KINOSHITA ${ }^{1)}$ and Toshio NUKADA ${ }^{1}$ ) \\ 1)Microbiology Division, Equine Research Institute, Japan Racing Association, Shimotsuke, Tochigi 329-0412, \\ Japan
}

\section{J. Vet. Med. Sci. \\ 81(9): 1234-1237, 2019}

doi: 10.1292/jvms.19-0192

Received: 8 April 2019

Accepted: 27 June 2019

Advanced Epub: 11 July 2019

\begin{abstract}
Actinobacillus species are known to be pathogenic to horses. To clarify etiological agents of actinobacillosis in Japanese adult horses, 27 isolates from Japanese Thoroughbred racehorses putatively identified by matrix-assisted laser desorption ionization time-of-flight mass spectrometry as Actinobacillus were further identified by PCR of the A. equuli toxin gene, by CAMP test, and by $16 \mathrm{~S}$ rRNA sequencing analysis. Actinobacillus equuli subsp. haemolyticus was isolated most frequently $(16 / 27)$ and was related to respiratory infections. Actinobacillus equuli subsp. equuli (4/27) was isolated from chronic cases or concomitant with other bacterial infections. The remainder were A. pleuropneumoniae, unclassified Actinobacillus species and Pasteurella caballi. Actinobacillus equuli including subsp. haemolyticus and subsp. equuli were the species most frequently isolated from equine actinobacillosis in Japan.

KEY WORDS: $16 \mathrm{~S}$ rRNA, Actinobacillus, matrix-assisted laser desorption ionization-time-of-flight mass spectrometry (MALDI-TOF MS), repeats-in-toxin (RTX), Thoroughbred
\end{abstract}

Actinobacillus species are Gram-negative rod-shaped bacteria classified in the family Pasteurellaceae [17]. Although Actinobacillus species naturally reside in the mucous membranes of the respiratory tract and genitourinary tract of healthy animals, some species are thought to cause disease when they invade tissues following local trauma [17]. Actinobacillus pleuropneumoniae (A. pleuropneumoniae) in particular is known to be highly contagious and to cause fibro-hemorrhagic necrotizing pneumonia in pigs [21]. Actinobacillus species were known to be associated specific hosts, and phenotypic identification of Actinobacillus at species level is difficult without information on host of isolation [4].

Among Actinobacillus species in horses, the most frequently isolated are Actinobacillus equuli. (A. equuli) subspecies (subsp.) equuli or A. equuli subsp. haemolyticus; Actinobacillus lignieresii (A. lignieresii) and A. pleuropneumoniae are also occasionally isolated [15], although A. pleuropneumoniae might be misidentified since the isolates were identified only by phenotypic characteristics and is exclusively isolated from pigs [3]. These organisms are associated with various clinical manifestations in horses including respiratory infections, septicemia, metritis, mastitis, arthritis, endocarditis, meningitis, or stillbirth [14]. Actinobacillus equuli subsp. equuli is the etiological agent of "sleepy foal disease" which is an acute (sometimes chronic) type of fatal septicemia in neonatal foals [10]. Actinobacillus equuli subsp. haemolyticus has been reported to have caused fatal pulmonary hemorrhage in an adult horse [19]. Systemic actinobacillosis is thought to occur mainly in neonatal foals because of failure of transfer of passive immunity and conditions in the birthing environment [23]. Adult horses are rarely infected with Actinobacillus species unless some other disease or predisposing factor is present [22]. Although, Thoroughbred racehorses are bred under stresses including strict training, race or long transportation, for horses an etiological agent of actinobacillosis in this population is still not clear.

Toxins belonging to the repeats-in-toxin (RTX) family are produced by some species of Actinobacillus, and they exert hemolytic and cytotoxic activity $[1,12]$. Actinobacillus equuli subsp. haemolyticus can be discriminated from A. equuli subsp. equuli by the presence of $A$. equuli toxin (Aqx), a member of the RTX toxin family, which is encoded by the aqx gene [14]. Furthermore, other Actinobacillus species are characterized by the existence of toxins of the RTX family [14]. Actinobacillus pleuropneumoniae RTX-toxin I (Apx I), Apx II, Apx III and ApxIV, which are encoded on polycistronic operons apxICABD, apxIICA, apxIIICABD and apxIVA respectively, play a role in the pathogenicity of $A$. pleuropneumoniae [20]. PCR assays based on apx genes have been used for identification and typing of A. pleuropneumoniae strains [25]. Production of Apx I and Apx II has been detected in A. suis [11], and A. lignieresii has apxICABD without the canonical promotor sequences and lacks expression of Apx I [20]. Analyzing these operons would help to identify Actinobacillus species. 
Table 1. Source of isolates used in this study and results of identification

\begin{tabular}{|c|c|c|c|c|c|c|c|}
\hline $\begin{array}{l}\text { Strain } \\
\text { No. }\end{array}$ & $\begin{array}{c}\text { Year of } \\
\text { isolation }\end{array}$ & $\begin{array}{l}\text { Age of } \\
\text { host }\end{array}$ & Source & MALDI-TOF MS identification & Aqx & $\begin{array}{l}\text { CAMP } \\
\text { test }\end{array}$ & Final identification \\
\hline NE-1 & 1996 & 4 & Guttural pouch infection & Actinobacillus equuli & + & + & Actinobacillus equuli subsp. haemolyticus \\
\hline NE-26 & 2000 & 2 & Shipping fever & Actinobacillus equuli & + & + & Actinobacillus equuli subsp. haemolyticus \\
\hline NE-28 & 2000 & 2 & Shipping fever & Actinobacillus equuli & + & + & Actinobacillus equuli subsp. haemolyticus \\
\hline NE-29 & 2000 & 2 & Shipping fever & Actinobacillus suis & + & + & Actinobacillus equuli subsp. haemolyticus \\
\hline NE-60 & 2000 & 2 & Shipping fever & [Actinobacillus] rossii & + & - & Actinobacillus equuli subsp. haemolyticus \\
\hline NE-72 & 2000 & 3 & Pneumonia & Actinobacillus equuli & + & + & Actinobacillus equuli subsp. haemolyticus \\
\hline NE-75 & 2000 & 3 & Pneumonia & Actinobacillus equuli & + & + & Actinobacillus equuli subsp. haemolyticus \\
\hline NE-77 & 2000 & 3 & Pneumonia & Actinobacillus. equuli & + & + & Actinobacillus equuli subsp. haemolyticus \\
\hline NE-87 & 2001 & 2 & Pneumonia & Actinobacillus equuli & + & + & Actinobacillus equuli subsp. haemolyticus \\
\hline NE-109 & 2002 & 2 & Guttural pouch infection & Actinobacillus equuli & + & + & Actinobacillus equuli subsp. haemolyticus \\
\hline NE-110 & 2002 & 3 & Pneumonia & Actinobacillus suis & + & + & Actinobacillus equuli subsp. haemolyticus \\
\hline NE-111 & 2002 & 3 & Pneumonia & Actinobacillus equuli & + & + & Actinobacillus equuli subsp. haemolyticus \\
\hline NE-178 & 2010 & 3 & Pneumonia & Actinobacillus suis & + & + & Actinobacillus equuli subsp. haemolyticus \\
\hline NE-186 & 2011 & 4 & Pneumonia & Actinobacillus equuli & + & + & Actinobacillus equuli subsp. haemolyticus \\
\hline NE-202 & 2013 & 3 & Pneumonia & Actinobacillus suis & + & + & Actinobacillus equuli subsp. haemolyticus \\
\hline NE-238 & 2015 & 2 & Abscess & Actinobacillus equuli & + & + & Actinobacillus equuli subsp. haemolyticus \\
\hline NE-134a) & 2004 & 3 & Thrombosis & Actinobacillus equuli & - & + & Actinobacillus equuli subsp. equuli \\
\hline NE-155a) & 2008 & 4 & Phlebitis & Actinobacillus equuli & - & - & Actinobacillus equuli subsp. equuli \\
\hline NE-185a) & 2011 & 3 & Endocarditis & Actinobacillus equuli & - & + & Actinobacillus equuli subsp. equuli \\
\hline NE-240 & 2015 & 2 & Stone bruise & Actinobacillus equuli & - & + & Actinobacillus equuli subsp. equuli \\
\hline NE-146a) & 2006 & 3 & Pneumonia & Actinobacillus pleuropneumoniae & - & - & Actinobacillus pleuropneumoniae \\
\hline NE-159 & 2009 & 3 & Lymphadenitis & Actinobacillus pleuropneumoniae & - & - & Actinobacillus pleuropneumoniae \\
\hline $\mathrm{NE}-2^{\mathrm{a})}$ & 1996 & 4 & Colitis & Actinobacillus pleuropneumoniae & - & - & Actinobacillus sp. \\
\hline NE-23 & 1999 & 3 & Pneumonia & Actinobacillus lignieresii & - & - & Actinobacillus sp. \\
\hline NE-144a) & 2005 & 3 & Pneumonia & [Actinobacillus] rossii & - & - & [Pasteurella] caballi \\
\hline NE-147 & 2006 & 3 & Pneumonia & [Actinobacillus] rossii & - & - & [Pasteurella] caballi \\
\hline NE-239 & 2015 & 2 & Stone bruise & [Actinobacillus] rossii & - & - & [Pasteurella] caballi \\
\hline
\end{tabular}

a) Isolated from a necropsied case. MALDI-TOF MS, matrix-assisted laser desorption ionization-time-of-flight mass spectrometry; Aqx, A. equuli toxin.

To clarify etiological agents of actinobacillosis in adult race horses, we characterized Actinobacillus species isolated from Japanese Thoroughbred racehorses.

The 27 bacterial isolates used in this study were obtained from 27 Thoroughbred racehorses ( 9 were 2 years old, 14 were 3 years old and 4 were 4 years old) between 1996 and 2015 (Table 1) and were kept at $-80^{\circ} \mathrm{C}$ until use. Of the 27 cases, 6 cases (NE-2, NE-134, NE-144, NE-146, NE-155, and NE-185) were necropsied cases and the other 21 were clinical cases (Table 1).

The isolates were putatively identified as Actinobacillus species by matrix-assisted laser desorption ionization-time-of-flight mass spectrometry (MALDI-TOF MS) performed on a MALDI Biotyper (Bruker Japan, Yokohama, Japan) according to the manufacture's instruction with database version 8.0.0.0. The species listed under MALDI-TOF MS in Table 1 are the best-match species presented by MBT Compass software (Bruker Japan).

The isolates identified by MALDI-TOF MS were then examined by analysis of the 16S rRNA sequence analysis, RTX gene PCR and CAMP activity as follows. 16S rRNA sequencing analysis was performed in accordance with published methods $[8,9]$ by sequencing $1,500 \mathrm{bp}$ PCR products amplified with primers: 27F, 5'-AGAGTTTGATCMTGGCTCAG-3' and 1525r, 5'-AAGGAGGTGATCCAGCC-3'. The obtained sequences were compared with published 16S rRNA gene sequences by using EZbiocloud (https://www.ezbiocloud.net) [24] and identified according to the guidelines of the Clinical and Laboratory Standards Institute [6]. The isolates were identified in the species level when the obtained sequences matched the reference sequence of type strain with over $99.0 \%$ identity and greater than $0.8 \%$ separation from other species, or identified in the genus level when the sequences matched the reference or valid sequences with $97.0-98.9 \%$ identity. To determine the presence of the RTX gene, Aqx-specific PCR based on the aqx gene, or Apx I-IV-specific PCR based on the presence of apxI-IV genes were performed as described previously [1, 7, 25], using bacterial genomic DNAs extracted by InstaGene Matrix (Bio-Rad, Hercules, CA, U.S.A.). For analysis of CAMP activity, a CAMP test using a Staphylococcus aureus streak on a sheep blood agar plate was performed. The isolates were finally identified comprehensively according to the results of MALDI-TOF MS identification, 16S rRNA sequence analysis, PCR of RTX gene or CAMP test gene sequencing.

The final identification of each isolate is described in Table 1. Of the 27 isolates, $16(59.3 \%)$ were identified as $A$. equuli subsp. haemolyticus which were aqx-positive and 4 (14.8\%) were A. equuli subsp. equuli which were aqx-negative. Of the remaining isolates, 2 (7.4\%) were A. pleuropneumoniae, 2 (7.4\%) were identified as Actinobacillus species but unclassified at the species 
level and $3(11.1 \%)$ were Pasteurella caballi. With the 16S rRNA sequence analysis, all the isolates except for 3 isolates identified as [Pasteurella] caballi had high similarity with multiple Actinobacillus species or with under $99 \%$ idnetity. With regard to the MALDI-TOF MS identification shown in Table 1, the results for some isolates in this study were inconsistent from the final identification. Four isolates (NE-29, NE-110, NE-178, and NE-202) were identified as Actinobacillus suis (A. suis) with MALDITOF MS; however, they were identified as A. equuli subsp. haemolyticus in the final identification. Three isolates (NE-144, NE-147, and NE-239) were identified as Pasteurella caballi with 16S rRNA sequencing, even though they were identified as [Actinobacillus] rossii with MALDI-TOF MS. Although MALDI-TOF MS is reported to be able to discriminate Actinobacillus species except for closely related species such as subspecies of A. equuli [13], these conflicting results seem to reflect the limitation of MALDI-TOF MS identification in these genera. For identification of isolates in Pasteurellaceae, other identification methods would be needed together with MALDI-TOF MS identification.

Four isolates (NE-2, NE-23, NE-146, and NE-159) were not identifiable at the species level by $16 \mathrm{~S}$ rRNA sequencing analysis. With the 16S rRNA sequencing analysis, 3 isolates (NE-2, NE-146, and NE-159) had lower than 99\% identity with Actinobacillus species; NE-2 matched $A$. hominis with $97.6 \%$ identity, NE-146 matched $A$. lignieresii with $98.89 \%$ identity, and NE- 159 matched A. lignieresii with $98.6 \%$ identity. NE-23 had high percent identity with two different Actinobacillus species; matched A. lignieresii with $99.2 \%$ identity and $A$. pleuropneumoniae with $99.0 \%$ identity. As reported previously [4], 16S rRNA sequencing analysis might have some difficulty in identifying certain Actinobacillus species. With the PCR of RTX, four isolates were aqx-negative and only apxIBD-positive, which suggested that these isolates had the operons apxI. NE-146 and NE-159 were also apxIV-positive, therefore they were identified as A. pleuropneumoniae. Further studied are needed for final identification of the strains that could not be identified in this study.

Of the 16 isolates identified as A. equuli subsp. haemolyticus, 9 isolates were isolated from cases with pneumonia, 4 were isolated from cases with shipping fever, 2 were isolated from cases with guttural pouch infection, and 1 was isolated from an abscess. In this study, A. equuli subsp. haemolyticus was the most frequently isolated from Thoroughbred racehorses in Actinobacillus species, and most of them were isolated from the respiratory tract. Recent study in the United States reported that A. equuli subsp. equuli was the most frequently isolated from adult horses [15], which showed different result from in this study. Although A. equuli subsp. haemolyticus is thought to be part of the normal flora of the equine oral cavity and has been isolated from tracheal washes [14], this species is also reported as a cause of pneumonia in young adult horses [3]. It is reported that the diseases caused by this species usually occur together with other factors, such as viral infection or stresses resulting from training [3], which is a situation that might have occurred in our cases.

Isolates identified as A. equuli subsp. equuli in this study were derived from horses with thrombosis, phlebitis, endocarditis, or stone bruise. Although A. equuli subsp. equuli is known to be a pathogen of newborn foals, it is often isolated from adult horses [15]. In this study, A. equuli subsp. equuli was isolated from 4 young adult horses, 3 of which were necropsied cases that might have had another chronic disease or bacterial infection before infection with A. equuli subsp. equuli. The case from which NE-134 was isolated had been treated earlier for colitis before finally being euthanized because of his poor condition. During the necropsy, not only A. equuli subsp. equuli but also Escherichia coli, Staphylococcus xylosus, Staphylococcus aureus, and Streptococcus equi subsp. zooepidemicus were isolated from the thrombus (data not shown). The other 2 necropsied cases were euthanized because of poor condition following acute laminitis (case of NE-155) or endocarditis (case of NE-185), and A. equuli subsp. equuli was the sole bacterium isolated from these 2 cases. In adult horses, A. equuli infections occur rarely or are limited to cases with some other concomitant disease or predisposing factor $[18,22]$. Our cases from which A. equuli subsp. equuli was isolated might each have suffered from another disease before Actinobacillus infection.

Actinobacillus species that produce RTX toxin show co-hemolytic (CAMP) activity towards erythrocytes [12]; however, some of the isolates in this study showed a contradiction between the results of the PCR of aqx analysis and the CAMP tests. One of the aqx-positive isolates (NE-60) did not show CAMP activity, and 3 isolates (NE-134, NE-185, and NE-240) were CAMP positive without possessing the aqx gene. The same phenotype has been reported for an Actinobacillus equuli strain isolated from a rabbit, in which there was a positive reaction in the CAMP test but where the aqx gene was not detected by PCR because of a DNA mutation in the primer binding site [16]. The isolates in our study might need further research to elucidate the reason for contradiction between $a q x$ PCR and CAMP test in this study. Furthermore, aqx-positive CAMP test-negative isolate (NE-60), which was identified as [A.] rossi with MALDI-TOF MS, might be the variant of A. equuli subsp. haemolyticus.

Two isolates were identified as A. pleuropneumoniae (NE-146 and NE-159) and two isolates (NE-2 and NE-23) were unclassified Actinobacillus species, and there was no consistency about their source or year of isolation. Even though these 4 isolates were not able to be identified by $16 \mathrm{~S}$ rRNA sequence analysis, 3 of them (NE-23, NE-146 and NE-159) had high similarity with $A$. lignieresii or A. pleuropneumoniae. A. lignieresii or A. pleuropneumoniae-like species have previously been isolated from horses: A. lignieresii has been isolated from the oral cavity of horses [5], and is reported to be a pathogen of equine cellulitis [2], and A. pleuropneumoniae-like species has been isolated from barren horses and horses with osteomyelitis [15]. In order to accurately discuss the pathogenicity of these Actinobacillus species, it will be necessary to isolate them from more cases.

In this study, it was clarified that $A$. equuli including subsp. haemolyticus and subsp. equuli was the primary cause of actinobacillosis in Thoroughbred racehorses in Japan. Each of the 2 subspecies showed different clinical states respectively.

ACKNOWLEDGMENTS. We thank our lab staff, Ms. M. Tezuka and Ms. F. Yomogida, for their technical support. 


\section{REFERENCES}

1. Berthoud, H., Frey, J. and Kuhnert, P. 2002. Characterization of Aqx and its operon: the hemolytic RTX determinant of Actinobacillus equuli. Vet. Microbiol. 87: 159-174. [Medline] [CrossRef]

2. Carmalt, J. L., Baptiste, K. E. and Chirino-Trejo, J. M. 1999. Actinobacillus lignieresii infection in two horses. J. Am. Vet. Med. Assoc. 215: 826-828, 796. [Medline]

3. Carr, E. A., Carlson, G. P., Wilson, W. D. and Read, D. H. 1997. Acute hemorrhagic pulmonary infarction and necrotizing pneumonia in horses: 21 cases (1967-1993). J. Am. Vet. Med. Assoc. 210: 1774-1778. [Medline]

4. Christensen, H. and Bisgaard, M. 2004. Revised definition of Actinobacillus sensu stricto isolated from animals. A review with special emphasis on diagnosis. Vet. Microbiol. 99: 13-30. [Medline] [CrossRef]

5. Christensen, H., Bisgaard, M. and Olsen, J. E. 2002. Reclassification of equine isolates previously reported as Actinobacillus equuli, variants of $A$. equuli, Actinobacillus suis or Bisgaard taxon 11 and proposal of A. equuli subsp. equuli subsp. nov. and A. equuli subsp. haemolyticus subsp. nov. Int. J. Syst. Evol. Microbiol. 52: 1569-1576. [Medline]

6. CLSI 2008. Interpretive criteria for identification of bacteria and fungi by DNA target sequencing; approved guideline. CLSI document MM18-A. Clinical and Laboratory Standards Institute, Wayne.

7. Frey, J., Beck, M., van den Bosch, J. F., Segers, R. P. and Nicolet, J. 1995. Development of an efficient PCR method for toxin typing of Actinobacillus pleuropneumoniae strains. Mol. Cell. Probes 9: 277-282. [Medline] [CrossRef]

8. Hiraishi, A. 1992. Direct automated sequencing of $16 \mathrm{~S}$ rDNA amplified by polymerase chain reaction from bacterial cultures without DNA purification. Lett. Appl. Microbiol. 15: 210-213. [Medline] [CrossRef]

9. Hiraishi, A., Shin, Y. K., Ueda, Y. and Sugiyama, J. 1994. Automated sequencing of PCR-amplified 16S rDNA on 'Hydrolink' gels. J. Microbiol. Methods 19: 145-154. [CrossRef]

10. Huang, B. F., Kropinski, A. M., Bujold, A. R. and MacInnes, J. I. 2015. Complete genome sequence of Actinobacillus equuli subspecies equuli ATCC 19392'. Stand. Genomic Sci. 10: 32. [Medline]

11. Kamp, E. M., Vermeulen, T. M. M., Smits, M. A. and Haagsma, J. 1994. Production of Apx toxins by field strains of Actinobacillus pleuropneumoniae and Actinobacillus suis. Infect. Immun. 62: 4063-4065. [Medline]

12. Kuhnert, P., Berthoud, H., Straub, R. and Frey, J. 2003. Host cell specific activity of RTX toxins from haemolytic Actinobacillus equuli and Actinobacillus suis. Vet. Microbiol. 92: 161-167. [Medline] [CrossRef]

13. Kuhnert, P., Bisgaard, M., Korczak, B. M., Schwendener, S., Christensen, H. and Frey, J. 2012. Identification of animal Pasteurellaceae by MALDITOF mass spectrometry. J. Microbiol. Methods 89: 1-7. [Medline] [CrossRef]

14. Kuhnert, P., Berthoud, H., Christensen, H., Bisgaard, M. and Frey, J. 2003. Phylogenetic relationship of equine Actinobacillus species and distribution of RTX toxin genes among clusters. Vet. Res. 34: 353-359. [Medline] [CrossRef]

15. Layman, Q. D., Rezabek, G. B., Ramachandran, A., Love, B. C. and Confer, A. W. 2014. A retrospective study of equine actinobacillosis cases: 1999-2011. J. Vet. Diagn. Invest. 26: 365-375. [Medline] [CrossRef]

16. Moyaert, H., Decostere, A., Baele, M., Hermans, K., Tavernier, P., Chiers, K. and Haesebrouck, F. 2007. An unusual Actinobacillus equuli strain isolated from a rabbit with Tyzzer's disease. Vet. Microbiol. 124: 184-186. [Medline] [CrossRef]

17. Mutters, R. 1999. Actinobacillus, Capnocytophaga, Eikenella, Kingella, and other fastidious or rarely encountered Gram-negative rods. pp. 561-571. In: Manal of Clincal Microbiology, 7th ed. (Murray, R.P. and Baron, E. J. eds.), American Society for Microbiology, Washington, D.C.

18. Patterson-Kane, J. C., Donahue, J. M. and Harrison, L. R. 2001. Septicemia and peritonitis due to Actinobacillus equuli infection in an adult horse. Vet. Pathol. 38: 230-232. [Medline] [CrossRef]

19. Pusterla, N., Jones, M. E. B., Mohr, F. C., Higgins, J. K., Mapes, S., Jang, S. S., Samitz, E. M. and Byrne, B. A. 2008. Fatal pulmonary hemorrhage associated with RTX toxin producing Actinobacillus equuli subspecies haemolyticus infection in an adult horse. J. Vet. Diagn. Invest. 20: 118-121. [Medline] [CrossRef]

20. Schaller, A., Kuhnert, P., de la Puente-Redondo, V. A., Nicolet, J. and Frey, J. 2000. Apx toxins in Pasteurellaceae species from animals. Vet. Microbiol. 74: 365-376. [Medline] [CrossRef]

21. Sebunya, T. N. and Saunders, J. R. 1983. Haemophilus pleuropneumoniae infection in swine: a review. J. Am. Vet. Med. Assoc. 182: $1331-1337$. [Medline]

22. Sternberg, S. 2001. Specific immune response of mares and their newborn foals to Actinobacillus spp. present in the oral cavity. Acta Vet. Scand. 42: 237-242. [Medline] [CrossRef]

23. Stewart, A. J., Hinchcliff, K. W., Saville, W. J. A., Jose-Cunilleras, E., Hardy, J., Kohn, C. W., Reed, S. M. and Kowalski, J. J. 2002. Actinobacillus sp. bacteremia in foals: clinical signs and prognosis. J. Vet. Intern. Med. 16: 464-471. [Medline] [CrossRef]

24. Yoon, S. H., Ha, S. M., Kwon, S., Lim, J., Kim, Y., Seo, H. and Chun, J. 2017. Introducing EzBioCloud: a taxonomically united database of $16 \mathrm{~S}$ rRNA gene sequences and whole-genome assemblies. Int. J. Syst. Evol. Microbiol. 67: 1613-1617. [Medline] [CrossRef]

25. Zhou, L., Jones, S. C. P., Angen, Ø., Bossé, J. T., Nash, J. H. E., Frey, J., Zhou, R., Chen, H. C., Kroll, J. S., Rycroft, A. N. and Langford, P. R. 2008. Multiplex PCR that can distinguish between immunologically cross- reactive serovar 3, 6, and 8 Actinobacillus pleuropneumoniae strains. $J$. Clin. Microbiol. 46: 800-803. [Medline] [CrossRef] 